\title{
One-trial excitatory backward conditioning as assessed by conditioned suppression of licking in rats: Concurrent observations of lick suppression and defensive behaviors
}

\author{
JOHN J. B. AYRES, CHRISTOPHER HADDAD, and MELODY ALBERT \\ University of Massachusetts, Amherst, Massachusetts
}

\begin{abstract}
Twenty eight male albino rats were given a single 4-sec 1-mA electric-grid-shock unconditioned stimulus (US). In the same session they received two 12-sec conditioned stimuli (CSs). One CS (explicitly unpaired) terminated $180 \mathrm{sec}$ before the US began; the other (backward paired) began immediately after the US terminated. The CSs used were a 1000-Hz 85-dB tone and an 84-dB click; their roles were counterbalanced. Over the next 2 days, each CS was presented for 2 min while the rats drank from a water bottle. The backward-paired CS was found to suppress licking more than the explicitly unpaired CS. This suppression was accompanied by an increase in defensive behavior (freezing and freeze/nod) and by a decrease in other activity. The suppression did not seem to be due to a maintained or enhanced CS-orienting response reflex, nor could it be attributed to an adventitiously reinforced interfering operant. The results support the presumption made in previous reports that the lick suppression evoked by a backward CS reflected onetrial backward excitatory fear conditioning.
\end{abstract}

The topic of backward conditioning has been controversial since the time of Pavlov. The history of that controversy is so well known that it needs no recitation. In recent times, the controversy has continued unabated. For example, in concluding a recent review article on backward conditioning, Spetch, Wilkie, and Pinel (1981) stated that "the empirical evidence for its existence can no longer be ignored. Thus the time for disputing whether backward conditioning is possible is past; it is time instead for systematic exploration of the variables that affect the magnitude and duration of the effect' (p. 174). By way of contrast, an even more recent review (Hall, 1984) concluded that "when only traditional Pavlovian conditioning studies are examined, the experimental findings suggest that UCS-CS trials [the backward conditioning procedure] will result in inhibition"' (p. 163).

The fact that these two reviews reached different conclusions is due in part to a disagreement about what kinds of studies should be reviewed. Spetch et al. leaned heavily on studies that used the conditioned suppression procedure. In that procedure, a conditioned stimulus (CS), such as a light or tone, previously paired with an aversive unconditioned stimulus (US), such as an electric shock, is presented to an animal working for some reward. The suppression in the rate of that rewarded behavior is taken as an index of the CS's conditioned strength. Hall (1984)

This work was supported by Grant BNS 85-00277 from the National Science Foundation to the first author. We wish to thank Michael S. Fanselow and Terry L. DeVietti for their critical reading of an earlier draft of the manuscript. Requests for reprints should be addressed to John J. B. Ayres, Department of Psychology, Middlesex House, University of Massachusetts, Amherst, MA 01003. excluded such studies from his review on two grounds. First, he pointed to a number of criticisms that had previously been made about the typical measure of suppression-the so-called "suppression ratio." Second, he alluded to a comment made by Gormezano and Kehoe (1975). Those authors had raised "the methodological possibility that instrumental responses are adventitiously acquired to the CS during the classical-conditioning phase, and these IRs [instrumental responses] when evoked by the CS in the test phase operate to modulate the instrumental target response" (p. 162). Each of Hall's criticisms deserves comment.

First, with regard to the suppression ratio, although we might like to defend it, to do so here would be a digression. We need only note that at least one of the conditioned suppression studies that provided evidence for backward excitatory conditioning, accepted by Spetch et al. (1981) but ignored by Hall (1984), did not use suppression ratios and is not subject to any of the criticisms leveled at that measure (Mahoney \& Ayres, 1976). The results of that study have since been replicated and extended (Burkhardt, 1980; Shurtleff \& Ayres, 1981).

Second, with regard to the comment by Gormezano and Kehoe (1975), it is true that during a Pavlovian forward conditioning procedure, a response that occurred during a CS might be adventitiously reinforced by the US or by its termination. If such a response were to be evoked by the CS on test, it could indeed conceivably cause a suppression of ongoing behavior. The implication would then be that the conditioned suppression reflected adventitious operant conditioning rather than Pavlovian conditioning. Such an account of conditioned suppression, however, 
seems extremely unlikely. Direct observations of rats in the presence of auditory CSs previously paired with shock USs usually reveals that the CS elicits freezing (e.g., Bouton \& Bolles, 1980; Fanselow \& Bolles, 1979; Sigmundi, Bouton, \& Bolles, 1980). Moreover, the amount of such freezing has been shown to be highly correlated with the degree of suppression evoked by the CS (Ayres \& Vigorito, 1984; Bouton \& Bolles, 1980; van Willigen, Emmett, Cote, \& Ayres, 1987). In contrast, on the first conditioning trial, freezing occurs only rarely (e.g., van Willigen et al., 1987); why, then, should either the US or its termination reinforce freezing more than other behaviors with a higher operant level? Furthermore, even if freezing or some approximation to it occurred more often during the conditioning trial, the possibility of adventitiously reinforcing that freezing would seem remote because explicit attempts to modify freezing using operant contingencies have led to the conclusion that freezing is not an operant, but is, rather, a respondent (Bolles \& Riley, 1973; Fanselow \& Lester, in press). The possibility of adventitious reinforcement seems even more remote in backward conditioning procedures, especially one-trial procedures such as those of Mahoney and Ayres (1976), Burkhardt (1980), and Shurtleff and Ayres (1981). When a CS does not begin until the US terminates, no event is programmed during the CS or after it. What then could serve as the adventitious reinforcer for the putatively interfering instrumental response? And, if there were some unknown, unprogrammed chance event during or after the CS that could serve as an operant reinforcer of responses occurring during a backward-paired CS, why does the same sort of adventitious operant conditioning not occur during explicitly unpaired control CSs, which also have no programmed events either during or following their occurrence? Why do CSs that have been backward paired with shock evoke more suppression on test than do explicitly unpaired control CSs? The adventitious operant interpretation of suppression strains credulity on several counts.

For the reasons just cited, we are inclined to dismiss Hall's (1984) critique of the conditioned suppression evidence for excitatory backward fear conditioning. However, a potentially more serious problem has recently been raised by van Willigen et al. (1987). These writers noted that in the conditioned suppression studies purporting to demonstrate excitatory backward fear conditioning, no direct observations of the subjects' behavior were described. We do not know, then, whether the test CS evoked freezing. It is conceivable that the backward conditioning procedure did not condition fear to the CS at all. Indeed, van Willigen et al. suggested that a US in proximity to a CS (during a backward conditioning trial) might retard habituation of the CS-orienting reflex (OR) (cf. Lubow, Schnur, \& Rifkin, 1976) or even enhance that reflex (Holland, 1977, 1979a, 1979b, 1980a, 1980b). If, on test trials, a previously backward-paired CS were to evoke a stronger OR than an explicitly unpaired control CS, and if that OR interfered with ongoing behavior, then the backward CS would elicit more suppression. That suppression, however, should not be attributed to conditioned fear.

The ORs that occur to the CSs that have been used in conditioned suppression studies of backward excitatory conditioning most often involve rearing and other active behaviors (van Willigen et al., 1987). If we were to observe rats tested with a CS previously backward paired with a shock US, we could determine whether test suppression was accompanied by defensive behaviors, such as freezing, or by OR-like behaviors, such as rearing and other activity. We would be justified in concluding that backward excitatory fear conditioning had been demonstrated only if the test suppression were accompanied by the defensive type of behavior. The purpose of the present experiment was to perform such observations. The method used was a one-trial differential conditioning procedure (van Willigen et al., 1987), in which each rat received one CS backward paired with shock and a second CS explicitly unpaired. Later these CSs were presented while the rats were licking a water bottle, and the lick suppression and other behaviors that the CSs evoked were recorded. A tone and clicking stimulus were chosen as the two CSs, because pilot work using 32 rats in a onetrial forward conditioning procedure had demonstrated that both CSs elicited suppression and freezing (although the tone tended to elicit more of both) and that generalization between the stimuli was undetectable.

\section{METHOD}

\section{Subjects}

The subjects were 28 male albino rats from the Holtzman Company, Madison, Wisconsin. They were 117 days old at the start of the experiment and had previously undergone barpress training for food. They were housed individually in suspended stainless steel mesh cages in a continuously lighted room maintained at about $76^{\circ} \mathrm{F}$. They were fed Purina Rat Chow ad lib but were placed on a 23.5-h water-deprivation schedule 3 days before the start of the experiment. During the study, the rats were given access to water for $.5 \mathrm{~h}$ after each daily session.

\section{Apparatus}

One Gerbrands operant conditioning box with its bar slot and recessed dipper tray covered with metal plates was housed in a ventilated .61-m cube of $12.7-\mathrm{mm}$ plywood lined with acoustical tile. The inside dimensions of the box were $23.2 \times 20.3 \times 19.5 \mathrm{~cm}$. The end walls were aluminum, and the sides and top were clear Plexiglas. The floor was made of 18 stainless steel rods, $2 \mathrm{~mm}$ in diameter, mounted $1.3 \mathrm{~cm}$ apart. In one side wall was a $5.1 \times$ $1.3 \mathrm{~cm}$ horizontal slot that gave access to a stainless steel lick tube. The lick tube was attached to a bottle of tap water and to a GrasonStadler drinkometer (Model E4690A-1) that recorded licks. The tip of the lick tube was $1 \mathrm{~mm}$ outside the box, and the top half of that tip was above the top of the slot; this positioning of the tube prevented all but tongue contacts. A BRS Foringer audio generator (Model au-901) provided an 85-dB 1000-Hz tone CS and an 84-dB click CS, at 333 clicks/min, as measured on an oscilloscope. Both the tone and the clicks were presented through a 10-cm-diam speaker mounted on the box lid. A high-voltage, high-resistance shock source provided a 4-sec 1-mA grid-shock US scrambled through a relay sequencing scrambler (Hoffman \& Fleshler, 1962). 
Two 7.5-W 110-V houselights were lit throughout the experiment; one was $11 \mathrm{~cm}$ outside the box directly in front of the lick tube, and the other was $15 \mathrm{~cm}$ outside the far wall from the lick tube and $20 \mathrm{~cm}$ above the floor of the housing cube. The cube had a snug-fitting, portable door made of two sheets of clear Plexiglas screwed to either side of a $10-\mathrm{cm}$ wooden frame. This door permitted an unobstructed view of the rat and preserved the sound attenuation of the cube. A SONY model AVC 3200 video camera was used to film the rats' behaviors during CSs on conditioning and test days. An NCR personal computer presented all experimenal stimuli and recorded licking.

\section{Procedure}

On Days 1 through 4, each rat was given access to the water bottle in the test box. On each day, the 100th lick produced a 2 -min dummy CS (no change from the rat's view). One minute after the end of the dummy CS, the rat was removed from the box, returned to its home cage, and watered for $.5 \mathrm{~h}$. Behavioral measures in the test box included the time between licks 90 and 100 (called the preCS time) and the time between licks 100 and 110 (called the CS time).

On Day 5, the rats received a one-trial backward differential conditioning procedure. They were placed individually in the test box with no lick tube available. Each rat was given a 4-sec 1-mA grid shock that began $214 \mathrm{sec}$ into a 432 -sec session. For 14 rats, a $12-$ sec tone CS began immediately after shock termination (backward conditioning procedure) and a 12-sec click CS terminated $180 \mathrm{sec}$ before US onset (explicitly unpaired procedure). For 14 other rats, the relationship of the two CSs to the US was reversed.

On Days 6 and 7, the rats were treated just as on Days 1 through 4 except that a test CS replaced the dummy CS. On Day 6, half the rats received their backward-paired $C S$ and half received their explicitly unpaired CS. On Day 7, they received the opposite CS. Rats failing to make 110 licks by the time the CS ended were given a CS time of $120 \mathrm{sec}$ : However, in the next minute, the computer continued to record the latency to the 110 th lick. The addition of this latency to the CS time is called "total suppression time." For example, if the time between licks 100 and 110 were $125 \mathrm{sec}$, the CS time would be $120 \mathrm{sec}$ and the total suppression time would be $125 \mathrm{sec}$. The total suppression time could not exceed $180 \mathrm{sec}$. This measure was recorded to reduce possible ceiling effects on the CS times, effects found previously in similar experiments on forward conditioning (van Willigen et al., 1987).

In addition to the lick suppression measures, a number of other behavioral measures were taken. Paced by a $1 / \mathrm{sec}$ click of a cam timer located near his head, the second author scored each rat's behavior in each second of the CS. Three behavioral categories were scored: (1) freezing (F), defined, as in previous work (e.g., Ayres \& Vigorito, 1984; Bouton \& Bolles, 1980; van Willigen et al., 1987), as the absence of any movement except for that of the rat's sides required for breathing; (2) freeze/nod (FN), defined as behavior that looked like freezing except that it included movement of the rat's head (we assumed that this behavior was a weaker form of freezing); and (3) other $(\mathrm{O})$, defined as anything not in the first two categories. When scoring these behaviors, the experimenter did not know whether the CS had been backward paired or explicitly unpaired. The behavior of the rats was also videotaped, and at the conclusion of the experiment, we scored rearing $(R)$ behavior during the 12-sec explicitly unpaired CS in the conditioning phase and during the first 12 sec of each test CS. Paced by the $1 / \mathrm{sec}$ click of the timer, we counted $R$ whenever the click coincided with the position of the rat's head slightly more than halfway to the top of the box. This judgment could be easily made by fixating on a certain row of holes in the acoustical tile on the back wall of the housing cube.

\section{RESULTS AND DISCUSSION}

For consistency with the presentations of other laboratories (e.g., Kasprow, Cacheiro, Balaz, \& Miller, 1982; Marlin, 1983; cf. van Willigen et al., 1987), the pre-CS times and CS times were transformed to logs (base 10). These measures are shown in Figure 1. The two left bars show the results for the tone and click combined; each of these means is based on 14 tone scores and 14 click scores. The figure suggests that there was more lick suppression to the backward-paired CS than to the explicitly unpaired CS, and this impression was supported by a $t$ test for correlated samples $[t(27)=3.00, p<.01]$. This difference between $\log$ CS times was based on comparable pre-CS rates, as the log pre-CS times for backward and unpaired test CSs did not differ significantly $[t s(27)<1]$.

The middle two and right two bars in Figure 1 show the results separately for the tone and click CSs, respectively. Clearly, the tone produced more lick suppression than did the click and yielded the larger difference between backward-paired and unpaired conditions, but, for both stimuli, the backward-paired CS appeared to produce more lick suppression; $t$ tests for uncorrelated samples found the difference to be significant for the tone $[t(26)$ $=4.06, p<.0005]$ and, by a one-tailed test, for the click $[t(26)=1.84, p<.05]$. (Note that there was virtually no suppression to the explicitly unpaired click [Figure 1, rightmost bar]; the mean log pre-CS and CS times for the explicitly unpaired click were .353 and .358 , respectively, a difference too small to appear in the figure.) Again, the differences in $\log$ CS times for the backward-paired and

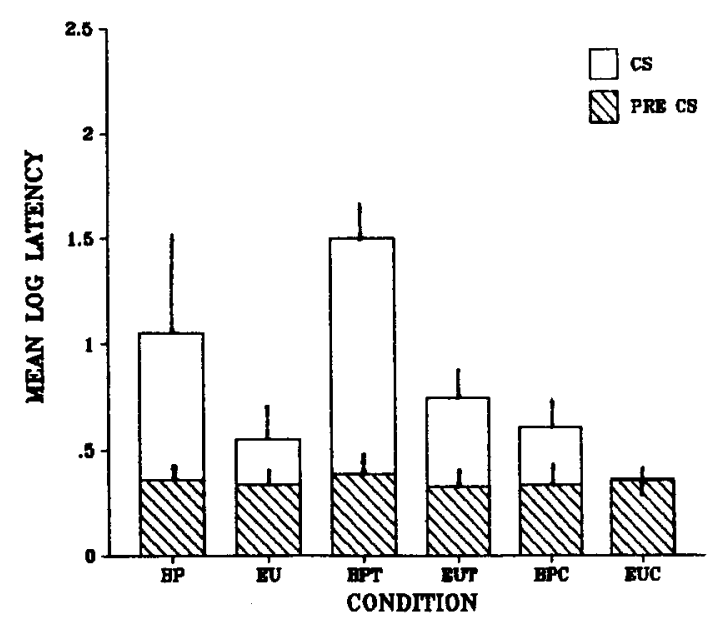

Figure 1. Mean log pre-CS times (striped part of bars) and log CS-times (entire bar) during test presentations of backward-paired (BP) and explicitly unpaired (EU) CSs. The two left bars show the results for the tone and click CSs combined. The middle two and right two bars show the results separately for the tone and click CSs, respectively. Vertical lines on top of the bars show one standard error of the mean. Abbreviations: BPT = backward-paired tone; EUC $=$ explicitly unpaired click. 
unpaired tones and clicks were based on comparable preCS rates, since, for the tone and click separately, no significant differences in the log pre-CS times were found $[t s(26)<1]$. Reanalyses of the lick suppression results using the logs of the total suppression-time measure (CS suppression plus post-CS suppression) did not alter any of the statistical decisions described above. In general, the lick-suppression data showed the same pattern that has been interpreted elsewhere as evidence for one-trial excitatory backward conditioning (Burkhardt, 1980; Mahoney \& Ayres, 1976; Shurtleff \& Ayres, 1981).

Figure 2 shows the behaviors that were observed directly. The results for the tone and click combined are shown in the left third of the figure. Correlated $t$ tests on those (combined) results found significantly more freeze/nod to the backward-paired CS than to the explicitly unpaired $\operatorname{CS}[t(27)=2.69, p<.02]$ and more "other" to the explicitly unpaired CS than to the backward-paired CS $[t(27)=2.15, p<.05]$. The tendency toward more freezing to the backward-paired CS was significant with a one-tailed test $[t(27)=1.77, p<.05]$. The middle third of the figure presents the results for the tone alone, and the right third presents the results for the click alone. Uncorrelated $t$ tests on these data found more freeze/nod to the backward-paired tone than to the unpaired tone $[t(26)=2.72, p<.02]$, more freezing to the backwardpaired click than to the unpaired click $[t(26)=2.23$, $p<.05$ ], and, by a one-tailed test, more "other" to the unpaired click than to the backward-paired click $[t(26)$ $=1.91, p<.05]$. At the conclusion of the experiment, the second author performed a reliability check of the measures shown in Figure 2. Selecting 7 subjects at random, he rescored their behavior using the videotapes. His

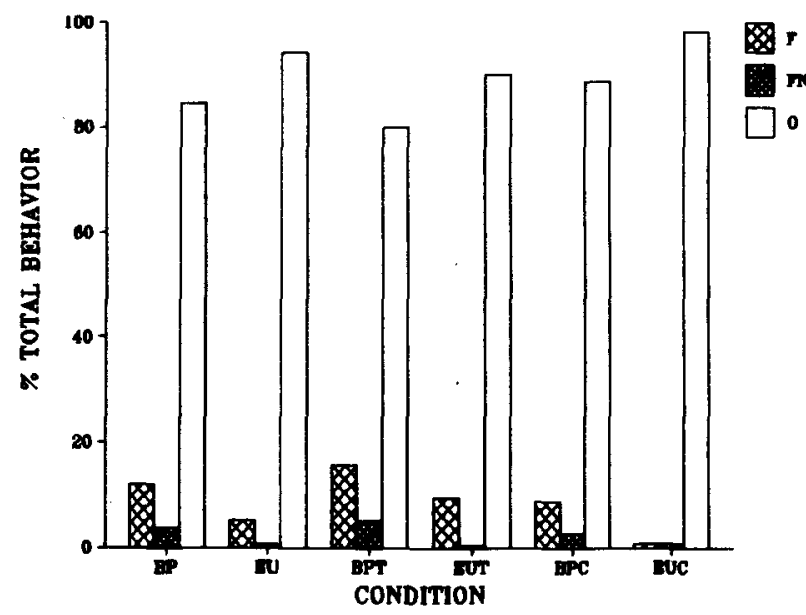

Figure 2. Mean percent of samples judged as freexing (F), freeze/nod (FN), and "other" (O) during test presentations of backward-paired (BP) and explicitly unpaired (EU) CSs. The left third of the ngure shows the results for the tone and click CSs combined. The middle and right thirds of the figure show the results separately for the tone and click CSs, respectively. Abbreviations: EUT $=$ explicitly unpaired tone; BPC = backward-paired click.
Table 1

Pearson Product Moment Correlations Among Conditioning Measures

\begin{tabular}{lrrrr}
\hline & \multicolumn{4}{c}{ Measure } \\
\cline { 2 - 5 } & CST & \multicolumn{1}{c}{ LCST } & F & FN \\
\hline CST & & & & \\
LCST & .88 & & & \\
F & .68 & .55 & & \\
FN & .53 & .40 & .61 & \\
O & -.70 & -.56 & -.98 & -.74 \\
\hline
\end{tabular}

Note-Abbreviations: CST $=$ CS time; $L C S T=\log$ CS time; F $=$ freezing; FN = freeze/nod (freezing with head movement); $\mathrm{O}=$ other (neither F nor FN).

second scoring agreed with his original scoring in $96 \%$ of the $\mathbf{8 4 0}$ observations remade.

Table 1 presents a matrix of correlation coefficients for the various measures of conditioning. All of the correlations were significant $(p s<.01)$. The correlations among the measures of lick suppression and defensive behavior (freeze and freeze/nod) are consistent with the hypothesis that these defensive behaviors are responsible for disrupting licking. The negative correlation between lick suppression and "other" argues against the possibility that suppression is due to the enhancement of a CSOR reflex, because the OR to auditory stimuli in our situation is typically an active behavior (van Willigen et al., 1987), and that would manifest itself in the form of higher "other" scores. The correlation between freeze and freeze/nod is consistent with the assumption that freeze/nod is a weaker form of freezing.

In general, the results in Figure 2 and in Table 1 support the idea that defensive behaviors evoked by the CS interfere with the ongoing lick behavior and are thus responsible, at least in part, for the conditioned lick suppression. The implication is that fear really is conditioned to the backward CS in the one-trial backward fear conditioning procedure, as has been presumed (Burkhardt, 1980; Mahoney \& Ayres, 1976; Shurtleff \& Ayres, 1981).

Although the foregoing suggests that defensive behaviors and not ORs are responsible for the lick suppression evoked by backward-paired CSs, we decided to examine the $O R$ issue more directly. At the conclusion of the experiment, we viewed the videotapes of conditioning to see exactly what our rats were doing during explicitly unpaired tones and clicks. We did not look at behavior to the backward-paired CSs because of the influence of the preceding shock. We were able to examine only 24 of the 28 rats during conditioning due to a flaw in one of the reels of videotape. We found that all 24 were extremely active during these trials and that all rats but one, at some time during the CS, stood fully erect and sniffed at the box lid under the speaker. We therefore decided to measure rearing as an index of the OR (see Procedure). Our measures revealed that the mean number of rearing responses during conditioning was 4.58 during the 12-sec tone and 4.25 during the 12 -sec click. In contrast, the mean number during the first $12 \mathrm{sec}$ of 
the backward-paired test CS was 1.42 for the tone and .17 for the click. The mean number during the first $12 \mathrm{sec}$ of the explicitly unpaired test CS was .75 for the tone and .25 for the click. Both backward-paired CSs were accompanied by significantly less rearing during testing than during conditioning $[t \mathrm{~s}(22)>3.60, p \mathrm{~s}<.002]$. The difference in rearing to backward-paired versus unpaired CSs during testing was not significant $[t \mathrm{t}(22)<1.40]$. It is clear that there was much less rearing to both backwardpaired and unpaired CSs in testing than there was during conditioning. This reduction of rearing during testing could have been due to a variety of factors: OR habituation during conditioning, crouching elicited by conditioned context cues during testing (Blanchard \& Blanchard, 1969), or the presence of a water tube in testing but not during conditioning. Regardless of which interpretation is correct, the main point is that greater lick suppression evoked by our backward CSs relative to our unpaired CSs cannot be explained in terms of a stronger OR evoked by the backward-paired CS, assuming rearing to be a valid measure of that OR. Rearing contributed little to lick suppression.

To assess an adventitious operant reinforcement account of the lick suppression evoked by the backward CS, we determined the percentage of observations scored as freezing and freeze/nod during the explicitly unpaired tone and click during conditioning. Since this information came from direct observation of the rats and not from the videotapes, data were available from all 28 rats. The mean percentage of observations judged as freezing was 5.9 to the tone and 1.2 to the click. None of the samples were judged as freeze/nod. Clearly, then, rearing and "other" activity were the dominant responses to the CSs during conditioning; and, if adventitious reinforcement were at work in the backward conditioning procedure, these would be the behaviors most likely to be reinforced, not freezing and freeze/nod. The results, then, are inconsistent with an adventitious operant reinforcement account (Gormezano \& Kehoe, 1975, as applied by Hall, 1984).

We have provided evidence that the lick suppression evoked by a CS from a one-trial backward aversive conditioning procedure reflects the conditioning of fear to the backward-paired CS. We have also provided evidence or argument that the lick suppression is caused neither by an interfering orienting response nor by an interfering adventitiously reinforced operant. In contrast, we have presented neither evidence nor argument that the lick suppression and defensive behaviors evoked by the backwardpaired CS reflect first-order and not second-order conditioning. The possibility that second-order conditioning might be involved was suggested many years ago by Mowrer and Aiken (1954). To our knowledge, their idea has never been explored. According to their scenario, when the US in a backward procedure is delivered, it conditions the background or context cues. The backward CS then occurs and is paired with those contextual cues and acquires excitatory strength by virtue of that pairing (cf.
Marlin, 1983). We hope to test this hypothesis in future research.

\section{REFERENCES}

AYres, J. J. B., \& Vigorito, M. (1984). Posttrial effects of presenting vs. omitting expected shock USs in the conditioned suppression procedure: Concurrent measurement of barpress suppression and freezing. Animal Learning \& Behavior, 12, 73-78.

Blanchard, R. J., \& BlanchaRD, D. C. (1969). Crouching as an index of fear. Joumal of Comparative \& Physiological Psychology, 67, 370-375.

Bolles, R. C., \& Riley, A. L. (1973). Freezing as an avoidance response: Another look at the operant-respondent distinction. Learning \& Motivation. 4, 268-275.

Bouton, M. E., \& Bolles, R. C. (1980). Conditoned fear assessed by freezing and by the suppression of three different baselines. Animal Learning \& Behavior, 8, 429-434.

BURKharDT, P. E. (1980). One-trial backward fear conditioning in rats as a function of US intensity. Bulletin of the Psychonomic Society, 15, 9-11.

Fanselow, M. S., \& Bolles, R. C. (1979). Triggering of the endorphin analgesic reaction by a cue previously associated with shock: Reversal by naloxone. Bulletin of the Psychonomic Society, 14, 88-90.

FANSELow, M. S., \& LESTER, L. S. (in press). A functional behavioristic approach to aversively motivated behavior: Predatory imminence as a determinant of the topography of defensive behavior. In R. C. Bolles \& M. D. Beecher (Eds.), Evolution and learning. Hillsdale, NJ: Erlbaum.

Gormezano, I., \& Kehoe, E. J. (1975). Classical conditioning: Some methodological-conceptual issues. In W. K. Estes (Ed.), Handbook of learning and cognitive processes. Vol. 2: Conditioning and behavior theory. Hillsdale, NJ: Erlbaum.

Hall, J. F. (1984). Backward conditioning in Pavlovian type studies: Reevaluation and present status. Pavlovian Journal of Biological Sciences, 19, 163-168.

Hoffman, H. S., \& Fleshler, M. (1962). A relay sequencing device for scrambling grid shock. Journal of the Experimental Analysis of Behavior, 5, 329-330.

Holland, P. C. (1977). Conditioned stimulus as a determinant of the form of the Pavlovian conditioned response. Journal of Experimental Psychology: Animal Behavior Processes, 3, 77-104.

Holland, P. C. (1979a). Differential effects of omission contingencies on various components of Pavlovian appetitive conditioned responding in rats. Journal of Experimental Psychology: Animal Behavior Processes, 5, 178-193.

Holland, P. C. (1979b). The effects of qualitative and quantitative variation in the US on individual components of Pavlovian appetitive conditioned behavior in rats. Animal Learning \& Behavior, 7, 424-432.

Holland, P. C. (1980a). CS-US interval as a determinant of the form of Pavlovian appetitive conditioned responses. Journal of Experimental Psychology: Animal Behavior Processes, 6, 155-174.

Holland, P. C. (1980b). Influence of visual conditioned stimulus characteristics on the form of Pavlovian appetitive conditioned responding in rats. Journal of Experimental Psychology: Animal Behavior Processes, 6, 81-97.

Kasprow, W. J., Cacheiro, H., Balaz, M. A., Miller, R. R. (1982). Reminder-induced recovery of associations to an overshadowed stimulus. Learning \& Motivation, 13, 155-166.

Lubow, R. E., Schnur, P., \& Rifkn, B. (1976). Latent inhibition and conditioned attention theory. Journal of Experimental Psychology: Animal Behavior Processes, 2, 163-174.

Mahoney, W. J., \& AYRES, J. J. B. (1976). One-trial simultaneous and backward fear conditioning as reflected in conditioned suppression of licking in rats. Animal Learning \& Behavior, 4, 357-362.

Marlin, N. A. (1983). Second-order conditioning using a contextual stimulus as S1. Animal Learning \& Behavior, 11, 290-294.

Mowrer, O. H., \& AIKEN, E. G. (1954). Contiguity vs. drive-reduction 
in conditioned fear: Temporal variations in conditioned and unconditioned stimulus. American Journal of Psychology, 67, 26-38.

ShURTLEFF, D., \& AYRes, J. J. B. (1981). One-trial backward excitatory fear conditioning in rats: Acquisition, retention, extinction, and spontaneous recovery. Animal Learning \& Behavior, 9, 65-74.

Sigmundi, R. A., Bouton, M. E., \& Bolles, R. C. (1980). Conditioned freezing in the rat as a function of shock intensity and CS modality. Bulletin of the Psychonomic Society, 15, 254-256.

SPetch, M. L., Wilkie, D. M., \& PINel, J. P. J. (1981). Backward conditioning: A reevaluation of the empirical evidence. Psychological Bulletin, 89, 163-175.

van Willigen, F., Emmett, J., Cote, D., Ayres, J. J. B. (1987) CS modality effects in one-trial backward and forward excitatory conditioning as assessed by conditioned suppression of licking in rats. Animal Learning \& Behavior, 15, 201-211.

(Manuscript received July 14, 1986;

revision accepted for publication December 15, 1986.) 